\title{
Correlation Inequalities and Equilibrium States
}

\author{
M. Fannes` and A. Verbeure \\ Instituut voor Theoretische Fysica, Universiteit Leuven, B-3030 Heverlee, Belgium
}

\begin{abstract}
For an infinite dynamical system, idealized as a von Neumann algebra acted upon by a time translation implemented by a Hamiltonian $H$, we characterize equilibrium states (KMS) by stationarity, a Bogoliubov-type inequality and continuous spectrum of $H$, except at zero.
\end{abstract}

\section{§1. Introduction}

The equilibrium states of a finite volume system in statistical mechanics is usually given by the Gibbs-ensembles.

To describe bona fide physical phenomena it is well known that one has to take the so-called thermodynamic limit i.e. the volume tending to infinity, of any of the Gibbs ensembles. These "limit Gibbs' states" have an interesting property, they satisfy the so-called KMS-condition $[1,2]$.

In [3] Roepstorff derived a stronger version of the Bogoliubov inequality [4] for Gibbs states (for KMS-states see [5]).

Let \langle\rangle$_{\beta H}$ denote the thermal average with respect to the Hamiltonian $H$ and the inverse temperature $\beta=1 / k T$. For any pair of observables $x, y$ the scalar product $(., .)_{\sim}$ is defined by:

$$
(x, y)_{\sim}=\frac{1}{\beta} \int_{0}^{\beta} d \lambda\left\langle\exp (\lambda H) x^{*} \exp (-\lambda H) y\right\rangle_{\beta H}
$$

(see also [6]). In [3] the following inequality is derived

$$
(x, x)_{\sim} \leqq\left[\left\langle x x^{*}\right\rangle_{\beta H}-\left\langle x^{*} x\right\rangle_{\beta H}\right] / \ln \left\langle x x^{*}\right\rangle_{\beta H} /\left\langle x^{*} x\right\rangle_{\beta H} .
$$

Of course we have not to insist on the importance of the Bogoliubov inequality and its stronger version in statistical mechanics (see e.g. [7]).

* Aangesteld Navorser NFWO, Belgium 
In this note we want to add an other argument in favor of the importance of the inequality (1). We prove that a basic concept like that of an equilibrium state is determined by the following three properties:

(i) Stationarity,

(ii) inequality (1),

(iii) spectral condition.

For the benefit of the reader we sketch here the argument for states on $\mathscr{B}\left(\mathbb{C}^{n}\right)$. We prove that (i) and (ii) imply the KMS-condition.

Let $H$ be the Hamiltonian, as operator on $\mathbb{C}^{n}$ with spectral resolution

$$
H=\sum_{i} \varepsilon_{i} E_{i}
$$

$\varepsilon_{i} \neq \varepsilon_{j},\left(E_{i}\right)$ spectral family of $H$.

We suppose that $\omega$ is a state on $\mathscr{B}\left(\mathbb{C}^{n}\right)$ satisfying conditions (i) and (ii). From (i)

$$
\omega(x)=\operatorname{Tr} \varrho x \quad x \in \mathscr{B}\left(\mathbb{C}^{n}\right),
$$

where $\varrho$ is a density matrix of the form

$$
\varrho=\sum_{i} R_{i},
$$

where

$$
0 \leqq R_{i} \leqq E_{i} \text { for all } i .
$$

Let $\mathscr{C}$ be the set partial isometries $V$ of rank one such that

$$
\begin{aligned}
& V^{*} V \leqq E_{i} \\
& V V^{*} \leqq E_{j}
\end{aligned}
$$

then from (ii) with $x=V$ one gets

$$
\frac{\exp \left(\varepsilon_{i}-\varepsilon_{j}\right)-1}{\varepsilon_{i}-\varepsilon_{j}} \leqq \frac{\omega\left(V V^{*}\right) / \omega\left(V^{*} V\right)-1}{\ln \omega\left(V V^{*}\right) / \omega\left(V^{*} V\right)}
$$

From the strict monotonicity of the function $f(x)=\frac{x-1}{\ln x}$ one gets

$$
\exp \left(\varepsilon_{i}-\varepsilon_{j}\right) \leqq \omega\left(V V^{*}\right) / \omega\left(V^{*} V\right)
$$

substituting $V$ by $V^{*}$ yields

$$
\exp \left(\varepsilon_{j}-\varepsilon_{i}\right) \leqq \omega\left(V^{*} V\right) / \omega\left(V V^{*}\right) .
$$

Hence

$$
\omega\left(V^{*} V\right) / \omega\left(V V^{*}\right)=\exp \left(\varepsilon_{j}-\varepsilon_{i}\right) .
$$

Hence

$$
\frac{\operatorname{Tr} R_{i} V^{*} V}{\operatorname{Tr} R_{j} V V^{*}}=\frac{\exp -\varepsilon_{i}}{\exp -\varepsilon_{j}}
$$


As this is true for any $V$ in $\mathscr{C}:$ there exists a constant $\alpha$ such that:

$$
\operatorname{Tr} R_{i} V^{*} V=\alpha \exp \left(-\varepsilon_{i}\right)
$$

and so

$$
R_{i}=\alpha \exp \left(+\varepsilon_{i}\right) E_{i} .
$$

From normalization : $\varrho=\exp (-H) / \operatorname{Tr} \exp (-H)$.

Remark that the original Bogoliubov inequality

$$
(x, x)_{\sim} \leqq 1 / 2\left\{\omega\left(x x^{*}\right)+\omega\left(x^{*} x\right)\right\}
$$

is not sufficient for determining the KMS-property.

This can be checked on $M_{2}$. Take

$$
H=\left(\begin{array}{ll}
0 & 0 \\
0 & 1
\end{array}\right) \quad \varrho=\left(\begin{array}{cc}
\alpha & 0 \\
0 & 1-\alpha
\end{array}\right) \quad 0 \leqq \alpha \leqq 1 .
$$

For $\alpha$ between

$$
(e-3 / 2) /(e-1) \leqq \alpha \leqq e / 2(e-1)
$$

the inequality is always satisfied, and the state need not to be KMS.

Therefore using the results of [3] and [5] we proved the equivalence of on the one hand the KMS-condition and on the other hand conditions (i) and (ii) i.e. stationarity and the inequality, which is an upper bound for the Duhamel two-point function.

We thank Professor E. Lieb for pointing out to us Ref. [8], where a different upper bound for the Duhamel two point function can be found. However it is unclear if this upper bound implies also the KMS-condition.

\section{§. The Main Theorem}

Let $\mathfrak{M}$ be a von Neumann-algebra on a Hilbert space $\mathscr{H}$ and $\left(\alpha_{t}\right)_{t \in R}$ such that $\alpha_{t}(x)$ $=\exp (i t H) \times \exp (-i t H)$ where $H$ is a self-adjoint operator on $\mathscr{H}$. Let $\Omega$ be a cyclic vector of $\mathscr{H}$ for $\mathfrak{M}$ and let $\omega$ be the corresponding vector state i.e. $\omega(x)=(\Omega, x \Omega)$; $x \in \mathfrak{M}$. Furthermore suppose that $\mathfrak{M} \Omega$ belongs to the domain $\mathscr{D}(\exp (-t H / 2))$ of $\exp (-t H / 2)$ for all $t \in[0,1]$. Then the following scalar product $(.,)_{\sim}$ :

$$
(x, y)_{\sim}=\int_{0}^{1} d t(\exp (-t H / 2) x \Omega, \exp (-t H / 2) y \Omega)
$$

is well defined on $\mathfrak{M}$.

Lemma 2.1. Suppose $\Omega$ cyclic and for all $x \in \mathfrak{M}$ :

$$
(x, x)_{\sim} \leqq \frac{\omega\left(x^{*} x\right)-\omega\left(x x^{*}\right)}{\ln \omega\left(x^{*} x\right)-\ln \omega\left(x x^{*}\right)} .
$$

Then $\Omega$ is separating. 
Proof. Suppose $x^{*} \Omega=0$ then $\omega\left(x x^{*}\right)=0$. Suppose $x \Omega \neq 0$ then $\omega\left(x^{*} x\right) \neq 0$ and the right hand side of $(+)$ vanishes; hence $(x, x)_{\sim}=0$.

As the integrand $\left(\exp \left(\frac{-t H}{2}\right) \times \Omega, \exp \left(\frac{-t H}{2}\right) \times \Omega\right)$ is continuous and positive

$$
\exp \left(\frac{-t H}{2}\right) x \Omega=0
$$

for all $t \in[0,1]$. For $t=0$ this yields $x \Omega=0$. Hence $x^{*} \Omega=0$ implies $x \Omega=0$. Therefore for all $y \in \mathfrak{M}$

$$
y x * \Omega=0,
$$

or $\left(x y^{*}\right)^{*} \Omega=0$ implies $x y^{*} \Omega=0$.

As $\Omega$ is cyclic for $\mathfrak{M}$, this implies that $x=0$. Q.E.D.

Lemma 2.2. Let (i) $\Omega$ cyclic and separating for $\mathfrak{M}$.

(ii) $\mathfrak{M} \Omega \subset \mathscr{D}\left(\exp \left(\frac{-H}{2}\right)\right)$.

(iii) $\mathfrak{K}$ linear, self-adjoint subspace of $\mathfrak{M}$ such that $\mathfrak{A} \Omega$ is dense in $\mathscr{H}$.

(iv) There exists a constant $C \geqq 1$ such that for all $x \in \mathfrak{K}$

$$
\begin{aligned}
& C^{-1}\left(\exp (-H / 2) \alpha^{*} \Omega, \exp (-H / 2) x^{*} \Omega\right) \leqq(x \Omega, x \Omega) \\
& \leqq C\left(\exp (-H / 2) x^{*} \Omega, \exp (-H / 2) x^{*} \Omega\right)
\end{aligned}
$$

then $(*)$ extends to all $y \in \mathfrak{M}$.

Proof. Define the operator $T$ on $\exp (-H / 2) \Re \Omega$ by

$$
T\left(\exp (-H / 2) x^{*} \Omega\right)=x \Omega \quad x \in \mathfrak{H} .
$$

By $(*) T$ is bounded by $\sqrt{C}$.

Now we prove the result by proving that there exists a closable extension $\tilde{T}$ of $T$. Define $\tilde{T}$ on $\exp (-H / 2) \mathfrak{M} \Omega$ by

$$
\tilde{T}\left(\exp (-H / 2) x^{*} \Omega\right)=x \Omega, \quad x \in \mathfrak{M} .
$$

$\tilde{T}$ is well defined $\left[\exp (-H / 2) x^{*} \Omega=0\right.$ implies $x^{*} \Omega=0$ and by (i) $\left.x \Omega=0\right]$ on a dense set $\exp (-H / 2) \mathfrak{M} \Omega$, as $\exp (-H / 2)$ is invertible.

We prove that the adjoint $\tilde{T}^{*}$ of $\tilde{T}$ is densily defined. First we prove that $\mathfrak{M}^{\prime} \Omega$ $\subseteq \mathscr{D}(\exp (H / 2))$, where $\mathfrak{M}^{\prime}$ is the commutant of $\mathfrak{M}$.

As $\exp (-H / 2)$ is a self-adjoint invertible operator, $\exp (-H / 2) \Re \Omega$ is dense in $\mathscr{H}$ and for all $y^{\prime} \in \mathfrak{M}{ }^{\prime}$ and $x \in \mathfrak{R}$ using $(*)$ :

$$
\begin{aligned}
& \left|\left(y^{\prime} \Omega, \exp (H / 2) \exp (-H / 2) x \Omega\right)\right|^{2} \\
& =\left|\left(x^{*} \Omega, y^{*} \Omega\right)\right|^{2} \leqq\left\|x^{*} \Omega\right\|^{2}\left\|y^{*} \Omega\right\|^{2} \\
& \leqq C\|\exp (-H / 2) x \Omega\|^{2}\left\|y^{*} \Omega\right\|^{2} .
\end{aligned}
$$

Hence

$y^{\prime} \Omega \in \mathscr{D}(\exp (H / 2))$. 
Define the operator $\tilde{T}^{+}$on $\mathfrak{M}^{\prime} \Omega$ [dense in $\mathscr{H}$, because of (i) by]:

$$
\tilde{T}^{+}\left(y^{\prime} \Omega\right)=\exp (H / 2) y^{\prime *} \Omega, \quad y^{\prime} \in \mathfrak{M}^{\prime} .
$$

$\tilde{T}^{+}$is well defined because $\Omega$ is cyclic for $\mathfrak{M}$.

Now $\tilde{T}^{*}$ is an extension of $\tilde{T}^{+}$, because for all $x \in \mathfrak{M}$ and $y^{\prime} \in \mathfrak{M}^{\prime}$ :

$$
\begin{aligned}
& \left(\tilde{T}^{+} y^{\prime} \Omega, \exp (-H / 2) x \Omega\right) \\
& =\left(\exp (H / 2) y^{\prime *} \Omega, \exp (-H / 2) x \Omega\right) \\
& =\left(y^{\prime} \Omega, x \Omega\right)=\left(x^{*} \Omega, y^{\prime} \Omega\right)=\left(\tilde{T} \exp (-H / 2) x \Omega, y^{\prime} \Omega\right) .
\end{aligned}
$$

Therefore the second inequality $(*)$ extends to all $x \in \mathfrak{M}$. Analogously for the other inequality. Q.E.D.

Theorem 2.3. Let $\omega$ and $\alpha_{t}$ be as above, if $\omega$ satisfies:

(i) $\omega$ is $\alpha_{t}$-invariant (stationnary state).

(ii) for all $x \in \mathfrak{M}$ :

$(x, x)_{\sim} \leqq\left[\omega\left(x x^{*}\right)-\omega\left(x^{*} x\right)\right] / \ln \omega\left(x x^{*}\right) / \omega\left(x^{*} x\right)$.

(iii) the spectrum of $H$ is continuous except for the point zero.

Then $\omega$ satisfies the KMS-condition for the evolution $\alpha_{t}$ at $\beta=1$, i.e. $\forall x, y \in \mathfrak{M}$ : $(\exp (-H / 2) y \Omega, \exp (-H / 2) x \Omega)=\left(x^{*} \Omega, y^{*} \Omega\right)$.

Proof. Suppose $E \in \operatorname{sp}(H) \cong R$ and $\delta>0$,

$$
\Delta=[E-\delta, E+\delta], \quad \Delta^{-}=[-E-\delta,-E+\delta]
$$

such that zero is not an endpoint of $\Delta$.

Let $H=\int \lambda d F(\lambda)$ be the spectral resolution of $H$ with spectral family $\{F(\lambda) / \lambda \in \mathbb{R}\} ; F_{\Delta}=\int_{\Delta} d F(\lambda)$.

Take any element $y \in \mathfrak{M}$ such that

$$
y \Omega \in F_{\Delta} \mathscr{H},
$$

then

$$
\begin{aligned}
& \int_{0}^{1} d \lambda(\exp (-t H / 2) y \Omega, \exp (-t H / 2) y \Omega) \\
& \geqq \int_{0}^{1} d \lambda \exp (-t(E+\delta))(y \Omega, y \Omega) \\
& =[(\exp (-(E+\delta))-1) /-(E+\delta)] \omega\left(y^{*} y\right) .
\end{aligned}
$$

By (ii):

$$
(\exp (-(E+\delta))-1) /-(E+\delta) \leqq \frac{\omega\left(y y^{*}\right) / \omega\left(y^{*} y\right)-1}{\ln \omega\left(y y^{*}\right) / \omega\left(y^{*} y\right)} .
$$

By the monotonicity of the function

$$
\lambda \rightarrow \frac{\lambda-1}{\ln \lambda}, \quad \lambda \in \mathbb{R}^{+}
$$


this yields

$$
\exp (-(E+\delta)) \leqq \omega\left(y y^{*}\right) / \omega\left(y^{*} y\right) .
$$

Also:

$$
(\exp (-H / 2) y \Omega, \exp (-H / 2) y \Omega) \leqq \exp (\delta-E) \omega\left(y^{*} y\right) .
$$

Hence

$$
\exp (-2 \delta)(\exp (-H / 2) y \Omega, \exp (-H / 2) y \Omega) \leqq \omega\left(y^{x}\right) .
$$

Analogously, remarking that $y^{*} \Omega \in F_{\Delta}-\mathscr{H}$ yields

$$
\omega\left(y y^{*}\right) \leqq(\exp (-H / 2) y \Omega, \exp (-H / 2) y \Omega \exp (2 \delta) .
$$

From (1) and (2):

$$
\begin{aligned}
& \exp (-2 \delta)(\exp (-H / 2) y \Omega, \exp (-H / 2) y \Omega) \leqq \omega\left(y y^{*}\right) \\
& \leqq(\exp (-H / 2) y \Omega, \exp (-H / 2) y \Omega) \exp (2 \delta)
\end{aligned}
$$

Let $\left\{\Delta_{k}^{n} / k \in Z\right\}$ be a partition of the real line such that

$$
\Delta_{k}^{n}=\left[\frac{2 k-1}{2 n}, \frac{2 k+1}{2 n}\right)
$$

for $n \in N$.

Let

$$
\mathfrak{S}_{k}^{n}=\left\{y \in \mathfrak{M} / y \Omega \in F_{\Delta_{k}^{n}} \mathscr{H}\right\}
$$

and $\mathfrak{H}^{n}$ be the linear span of the $\mathfrak{\Re}_{k}^{n}$ for all $k \in Z$. We prove that $\mathfrak{S}^{n} \Omega$ is dense in $\mathscr{H}$.

Take any $\psi \in F_{\Delta_{k}^{n}} \mathscr{H}$, for any $\varepsilon>0$, there exists an element $x \in \mathfrak{M}$ such that $\|\psi-x \Omega\|<\varepsilon$.

Take

$$
x(f)=\int f(t) \alpha_{t}(x) d t
$$

with $f \in L^{1}(R)$ and support of the Fourier transform $\hat{f}$ of $f$ in $\Delta_{k}^{n}$, then $x(f) \Omega \in F_{\Delta_{k}^{n}} \mathscr{H}$.

Because of condition (iii), it is furthermore possible to choose $f$ such that

$$
\left\|x(f) \Omega-F_{\Delta_{k}^{n}} x \Omega\right\|<\varepsilon .
$$

Then

$$
\left\|\psi-x_{(f)} \Omega\right\| \leqq\left\|\psi-F_{\Delta_{k}^{n} x} \Omega\right\|+\left\|F_{\Delta_{k}^{n} \alpha} \Omega-x(f) \Omega\right\|<2 \varepsilon
$$

proving that $\mathfrak{R}^{n} \Omega$ is dense in $\mathscr{H}$.

The inequalities (3) are easily extended to $\boldsymbol{R}^{n}$. Take

$$
x=\sum_{k=1}^{N} x_{k}, x_{k} \in \mathfrak{\Re}_{k}^{n}
$$


then

$$
\begin{aligned}
& \exp (-1 / n)(\exp (-H / 2) x \Omega, \exp (-H / 2) x \Omega) \\
& =\exp (-1 / n) \sum_{n}\left(\exp (-H / 2) x_{k} \Omega, \exp (-H / 2) x_{k} \Omega\right) \\
& \leqq \sum_{k}\left(x_{k}^{*} \Omega, x_{k}^{*} \Omega\right)=\left(x^{*} \Omega, x^{*} \Omega\right)
\end{aligned}
$$

and analogously for the second inequality.

Now we are in a position to use Lemma 2.2 yielding (3) for all $x \in \mathfrak{M}$. As this is true for all $n$ we get for all $x \in \mathfrak{M}$ :

$$
(\exp (-H / 2) x \Omega, \exp (-H / 2) x \Omega)=\left(x^{*} \Omega, x^{*} \Omega\right) .
$$

By polarization, for all $x$ and $y \in \mathfrak{M}$, we get

$$
(\exp (-H / 2) y \Omega, \exp (-H / 2) x \Omega)=\left(x^{*} \Omega, y^{*} \Omega\right)
$$

which is a particular form of the KMS-equation. Q.E.D.

\section{References}

1. Kubo, R.: J. Phys. Soc. Japan 12, 570 (1957);

Martin,P.C., J. Schwinger: Phys. Rev. 115, 1342 (1959)

2. Haag, R., Hugenholtz,N., Winnink, M.: Commun. math. Phys. 5, 215 (1967)

3. Roepstorff, G. : Commun. math. Phys. 46, 253 (1976); see also: Roepstorff, G.: A stronger version of Bogoliubov's inequality and the Heisenberg model. Preprint August 1976, TH Aachen, Germany

4. Bogoliubov, N.: Phys. Abh. S.U. 1, 229 (1962)

5. Naudts, J., Verbeure, A.: J. Math. Phys. 17, 419 (1976)

6. Naudts,J., Verbeure, A., Weder, R.: Commun. math. Phys. 44, 87 (1975)

7. Mermin, N., Wagner,H.: Phys. Rev. Lett. 17, 1133 (1969);

Dyson,F.J., Lieb,E.H., Simon, B.: Phys. Rev. Letters 37, 120 (1976);

Dyson, F.J., Lieb, E.H., Simon, B. : Phase transitions in quantum spin systems with isotropic and nonisotropic interactions. J. Stat. Phys. (to appear)

8. Bruch,L.W., Fortune,P.J., Berman, D.H.: J. Chem. Phys. 61, 2626 (1974), Appendix B

Communicated by E. Lieb

Received February 6, 1977 
